Volume 9, No.5, September - October 2020

International Journal of Advanced Trends in Computer Science and Engineering

Available Online at http://www.warse.org/IJATCSE/static/pdf/file/ijatcse260952020.pdf

https://doi.org/10.30534/ijatcse/2020/260952020

\title{
Modeling the Process of Filtration of Fluid and Protection of Groundwater from Ionic Pollutants
}

\author{
U.Saidov, T.Azamov, Y. Sultonov, Z.Ravshanov \\ Tashkent University of Information Technology, Uzbekistan, ravshanzade-09@mail.ru
}

\section{ABSTRACT}

The article deals with the problem associated with environmental protection, protection of groundwater from pollution sources, that is, the technology of ion-exchange filtration of liquid solutions from heavy ionic compounds emitted from production facilities and for a comprehensive study, a mathematical model and its approximate analytical solution describing a nonlinear system are presented. partial differential equations. In the work for the numerical integration of the problem, in order to suppress small-scale oscillations, a term of artificial viscosity is added with a certain numerical parameter - the coefficient of artificial viscosity, and the nonlinear terms of the equation are linearized by the Bellman-Kalaba quasi-linearization method. Since when filtering the suspension, the pores of the filter are filled with gel particles, the mathematical formulation of the problem takes into account the effect of ion exchange on the porosity and permeability of the ion exchange filter and gives the law of its change.

Key words: Mathematical model, approximate analytical solution, numerical algorithm, technological process of filtration, protection of groundwater.

\section{INTRODUCTION}

At present, threats to environmental safety are emerging in the world, in particular, the problems of shortage and pollution of water resources, provision of the population with drinking water, groundwater pollution from pollution sources, radiation pollution of certain territories, carbon dioxide emissions into the atmosphere, etc. from industrial facilities.

To solve the above problems and similar tasks, it is necessary to develop an effective tool, which can be used to conduct a comprehensive study, determine the main factors and their ranges of change depending on the operating modes of the filtering units and the disturbance acting on the technological process (TP) as a whole, with the aim of making managerial decision.

One of the most effective method for solving the problem is to develop a mathematical tool: a mathematical model (MM), a numerical algorithm and a software-instrumental complex, which I can use to carry out computational experiments on a computer when setting various values of the TP parameters and operating modes of filtering units, physical and mechanical properties of mixtures.

Many scientists were engaged in the problems of mathematical modeling of the process of filtration and separation of complex components of the suspension and they obtained significant theoretical and applied results.

In particular, the article [1] discusses the problem of deterioration of reservoir permeability in the bottomhole zone, as well as the problem of reducing water intake of injection wells due to clogging of pores and channels of the productive formation with suspended solids present in the water. The article presents the characteristics of the main wells and the dynamics of their parameters from the very beginning of their development of these wells. The author of the article proposes a new method for deep processing of the formed water, which ensures the capture of suspended solids in the reservoir.

In [2], a conservative numerical scheme was proposed for studying the process of filtration of a multiphase flow in a porous medium, where the characteristic size of the porous medium is from microns to millimeters. The numerical method is developed for a comprehensive study of the multiphase flow filtration process in porous media with complex interface motion, and the Navier - Stokes equations are discretized using the finite volume approach. In the paper, capillary forces are calculated using a peninsular surface force model, in which the transition region for capillary pressure is effectively limited to one mesh block. This new formulation, together with two new filtration methods developed to correct capillary forces, allows simulations at very low capillary numbers and avoids unphysical velocities. Capillary forces are implemented using a semi-implicit scheme that allows an increase in the time step size with a low number of capillaries, and the accuracy and conservatism of the numerical method is verified using several test examples that indicate the potential of the method for predicting multiphase flow processes.

In the article [3], the issues of taking into account the reverse effect of the process characteristics on the characteristics of the medium (coefficients of porosity, filtration, diffusion, mass transfer, etc.) are solved, using the example of liquid purification using magnetic and sorption filters. An algorithm is proposed for the 
numerical-asymptotic approximation of the solution of the corresponding model problem described by a system of nonlinear singular-perturbative differential equations of the "convection-diffusion-mass transfer" type. Corresponding ratios are effective in carrying out theoretical studies aimed at the "productivity" (in particular, optimization) of the parameters of the filtration process. In cases where convection and sorption components prevail over diffusion and desorption components, which occurs in the overwhelming majority of filtration plants. On this basis, a computer experiment was carried out. These results show the advantages of the proposed model over the classical one.

The article [4] considers the problem of identifying unknown parameters of the mathematical model of the technological process of an ion exchange filter. The developed mathematical model of the process consists of a material balance equation, an equation describing the kinetics of ion exchange for the nonequilibrium case and an equation for the isotherm of ion exchange. The material balance equation includes a nonlinear term that depends on the kinetics of ion exchange and several parameters of the research object. To determine the concentration of impurities at the outlet, the direct problem was solved, and then the inverse problem was formulated to find the parameters of the ion-exchange process in nonequilibrium conditions.

In work [5] the technological schemes of water purification are considered based on the alternation of two processes filtration and washing. The presence of multicomponent impurities in rinsing waters adversely affects the processes of their purification and leads to a significant deterioration in the quality of wastewater. The solution to the problem is achieved by separating wash water contaminants from the phase-dispersed state, which reduces the concentration of ferrous compounds and brings their concentration in the wash water to a level acceptable for discharge into treatment facilities or water bodies. The paper developed a mathematical model of the process of cleaning a liquid from multicomponent contaminants, taking into account the interaction of hydrodynamic parameters, changes in the hydraulic properties of the medium, taking into account the corresponding values of the concentration of contamination in the liquid and sediment. The influence of the filtration component on the porosity and the coefficient of separated particles in the sediment is considered. The time of the protective action of the layer of filtering material and the time of its regeneration were determined, taking into account the values of the concentration of contaminants in the water.

The article [6] considers some aspects of modeling the diffuse interface - incompressible flows, consisting of three immiscible components, without taking into account the phase transition. In diffuse interface methods, the evolution of the system is due to the minimization of free energy. The originality of this approach, based on the Kahn Hilliard model. To improve the three-phase flow model, the authors used the Cahn - Hilliard system and the NavierStokes equations, which take into account surface tensions and volumetric capillary forces. To solve the problem, the continuous domain of changing the variables is replaced by a grid, discretized in time and space. Various numerical results are presented for the case of checking the distribution of a lens between two phases, and mass transfer across the liquid-gas interface is investigated for their different values of density and viscosity.

In [7], a mathematical model was proposed for the adsorption of cephalosporin $\mathrm{C}$ (CPA) in a fixed bed column and a particle swarm optimization (PSO) algorithm for parameter estimation was first considered, which turned out to be a useful tool for parameter estimation in adsorption processes.

The article [8] proposes a transport model for the migration of a pollutant through mineral barriers in the presence of chemical-water gradients, which takes into account the flow of liquid and chemicals in the process of nonlinear diffusion. The resulting transport equations are solved by an asymptotic expansion based on the multiple time scale technique, and the steady state profile is calculated analytically.

In [9], the efficiency of application of vertical drainage elements with porous fibrous shells in high-speed filters with granular loading was substantiated with the help of theoretical studies. To study TP, a mathematical model was developed for parallel filtration of a low-concentration suspension through a granular and fibrous medium. On the basis of numerical experiments, significant parameters of the filter of an improved design are determined. Examples of calculations of rational values of the main parameters are shown.

Work [10] is devoted to the process of filtering drinking water using a filtering system of cartridges in order to check their hydraulic characteristics and, in particular, to assess the head loss. In these systems, the installation of filter cartridges can reduce the pressure level below the minimum limit recommended for uninterrupted operation of household appliances and seven different industrial filter cartridges have been studied and analyzed through a series of experiments. The analysis has shown that for typical operating flow rates in domestic installations, pressure drops across the filter can be on the order of several meters and has provided some useful information for selecting the right cartridge in low pressure distribution systems.

In [11], a phenomenological model of depth filtration is proposed. It combines the advection-dispersion equation with the equation of nonlinear multistage accumulation kinetics. The model includes dispersion and takes into account temporal and spatial changes in the porosity of the medium. It is assumed that at any place inside the column, a filter cake is first formed as an irreversible maturing layer, and then a reversible cake is formed during the working step. The latter continues until the deposit reaches 
the local maximum value, and then the filter breaks out. The equations are solved numerically using an explicit finite difference scheme.

The article [12] discusses the issues of water treatment in the development of oil fields with water flooding. It is known that the injected water contains oil residues and mechanical impurities that affect the permeability of the geological horizon. It is shown that these water impurities can enhance the clogging of rocks up to the complete clogging of pores and cracks. Thus, the issues of improving the quality of injected water aimed at improving reservoir properties have been studied from various angles. The results of the carried out test experiments are presented, as well as a detailed description of the developed equipment design. It has been established that the two-stage purification technology proposed in the article using a magnetic treatment device and a wastewater tank with a liquid hydrophobic layer will significantly improve the quality of injected water. The proposed water treatment plant seems to be very applicable, providing maximum effect with minimum cost of materials.

From the technology of filtration of the solution, it can be seen that at a constant pressure drop, the filtration rate decreases as the thickness of the sediment layer increases and its compression under the action of the hydraulic pressure formed in the column of the unit. If the suspension is supplied to the filter column by a piston pump, the filtration process occurs with a continuous increase in the pressure drop with a constant speed of its passage through the filter partition. If the slurry is supplied by a centrifugal pump, both the pressure drop and the filtration rate change continuously.

When filtering heavily contaminated, multicomponent liquid and ionized solutions, a sediment layer forms on the surface of the filtering partition and, under the action of the surface pressure force, the layer is repackaged and compressed. Compression of the sludge leads to an increase in hydraulic resistance and a decrease in the rate of filtration of the solution. To prevent compression, coagulants and folliculitis are added to finely dispersed suspensions, which contribute to the aggregation of fine particles and increase the porosity of the sediment layer.

Filters are used in technological cycles to purify and filter waste, waste water, industrial solutions, food products, pharmaceutical raw materials and other liquids from unwanted ionic concentrates, industrial waste. Usually, these solutions in their compositions, along with ionic impurities, contain gel particles of various dimensions and properties [13-16].

When filtering these solutions:

- particles in them become clogged in the pores of the filter, which leads to their swelling, as a result of which its throughput decreases;

- a layer of sediment is formed, as a result of which the laws of the flow of TP in general change; a liquid solution that undergoes filtration is not completely purified from unwanted ions, as a result of which it, getting into the environment, worsens the ecological state of the region.

\section{PROBLEM STATEMENT}

Taking into account the above, to analyze the functioning, study and determine the main parameters of the object and their ranges of change, taking into account the above factors, on the basis of the laws of hydrodynamics and kinetics of the process, a mathematical model has been developed [17-20]:

$$
\begin{aligned}
& \left\{\frac{\partial m n}{\partial t}+\frac{\partial u n}{\partial x}+\frac{\partial N}{\partial t}=\frac{\partial}{\partial x} D \frac{\partial \eta}{\partial x}+\frac{D \delta}{P} \frac{\partial P}{\partial x},\right. \\
& \frac{\partial N}{\partial t}=\beta\left(n-n^{\prime}\right), N=\frac{n^{\prime}}{a+b n^{\prime}} . \\
& \frac{\partial u}{\partial t}+u \frac{\partial u}{\partial x}=-\frac{1}{\rho} \frac{\partial P}{\partial x}+\frac{\mu}{\rho} \frac{\partial^{2} u}{\partial x^{2}}-\frac{\mu H u}{\rho H k_{0}(1-\delta)^{2}} ; \\
& \left\{\frac{\partial m \theta}{\partial t}+\frac{\partial u \theta}{\partial x}+\frac{\partial m \alpha}{\partial t}+\left(1-m_{0}\right) \frac{\partial m \delta}{\partial t}=0 ;\right. \\
& \frac{\partial \delta}{\partial t}=\lambda(\theta-\gamma \delta), \quad \theta=\frac{\alpha}{1-\delta}, \\
& m=m_{1}+\delta\left(m_{0}-m_{1}\right) \text {, }
\end{aligned}
$$

where $n$ and $N$ are the concentrations of the sorbed substance in the solution and sorbent, calculated per unit length of the sorption column; $u$ - filtration rate; $n^{\prime}$ - the concentration of ions in the solution, which is in equilibrium with the concentration; $N$; $P$ - pressure in the column of the unit; $D, D_{\delta}$ - coefficient of longitudinal diffusion and barodiffusion; $\theta$ and $\delta$ - concentration of suspended matter in a moving mixture and settling of particles in pores; $\alpha$ - concentration of suspended matter in suspension in the pores of the filter; $\gamma$ - dispersion coefficient; $\beta$ - effective rate constant of exchanging ions; $a, b$ - constant sorption isotherms; $m_{1}$ and $m_{2}$ - the initial porosity of the filter and the porosity of the settled mass; $k_{0}$ - filter permeability coefficient.

From the statement of the problem and the first equations of system (1) - (2), it can be seen that it is an equation of a hyperbolic type, and its numerical integration results in a small-scale oscillation, due to which round-off errors accumulate and its amplitude will grow over time.

Therefore, when numerically integrating such equations, an additional term of "artificial viscosity" is usually added with a certain numerical parameter - the coefficient of artificial viscosity. It should be emphasized here that the coefficient of "artificial viscosity" in each specific problem should be selected separately during the computational experiment. 


\section{SOLVING METHOD}

Methods for solving the problem. For the convenience of integration, we introduce dimensionless variables and instead of system (2) we obtain the following equations:

$$
\begin{aligned}
& \frac{\partial u}{\partial t}+u \frac{\partial u}{\partial x}=-E u \frac{\partial P}{\partial x}+\frac{1}{\operatorname{Re}} \frac{\partial^{2} u}{\partial x^{2}}- \\
& -\frac{u}{\operatorname{Re}_{1}(1-\delta)^{2}}+u \rho \frac{\partial \theta(1, t)}{\partial t} ; \\
& \frac{\partial m \theta}{\partial t}+\frac{\partial u \theta}{\partial x}+\frac{\partial m \alpha}{\partial t}+\left(1-m_{0}\right) \frac{\partial m \delta}{\partial t}=\frac{\mu_{0} \alpha_{\tau}}{x_{0}^{2}} \frac{\partial^{2} \theta}{\partial x^{2}} ; \\
& \frac{\partial \delta}{\partial t}=\lambda_{1}(\theta-\gamma \delta)
\end{aligned}
$$

and the boundary conditions of problem (3) - (5) are written in the form

$$
\left\{\begin{array}{l}
u=1, \theta=e^{-\lambda \gamma H_{0} x}, \delta=0, t=0 \\
\frac{\partial u}{\partial x}=\frac{H_{0}^{3}}{H k_{0}}\left[(E u / \operatorname{Re}) P-\frac{u}{(1-\delta)^{2}}\right], \theta=1, x=0 \\
\frac{\partial u}{\partial x}=0, \frac{\partial \theta}{\partial x}=\frac{m\left(1-m_{0}\right)}{u} \delta, x=1
\end{array}\right.
$$

where $\operatorname{Re}=\frac{\rho H_{0} u_{0}}{\mu}, \operatorname{Re}_{1}=\frac{\rho H k_{0} u_{0}}{\mu H_{0}^{2}}-$ Reynolds number, $E u=\frac{\rho_{0} H k_{0}}{H_{0} \mu}-$ Euler's number.

Problem (3) - (5) is solved numerically using the sweep method.

As mentioned above, as a result of filtering the suspension using a filtering overhead, the pores of the filter are filled with gel particles. Therefore, it is necessary to take into account the effect of ion exchange on the porosity and permeability of the ion exchange filter, which obey the laws

$$
m=m_{1}-\frac{N}{\rho_{c}}
$$

$k=A_{0} d^{2} k_{0} \frac{m^{3}}{(1-m)^{2}}=\frac{A_{1} k_{0}}{S_{0}^{2}} \frac{m^{3}}{(1-m)^{2}}$,

where $\rho_{c}$ - density of the sorbed substance in the solid phase: $d$ - particle diameter; $S_{0}$-specific surface area of a porous medium; $A_{0}$ и $A_{1}$ - permanent.
Then the balance equation for sorption in system (1) takes the form

$$
\frac{\partial\left(m_{1}-\frac{N}{\rho_{c}}\right) n}{\partial t}+\frac{\partial u n}{\partial x}+\frac{\partial N}{\partial t}=\frac{1}{P e} \frac{\partial}{\partial x} D \frac{\partial n}{\partial x}+\frac{D_{\delta}}{u_{0} n_{0} P} \frac{\partial P}{\partial x}
$$

We apply the quasilinearization method [5] to the first term of this equation and obtain

$$
\begin{aligned}
& \left(m_{1}-\frac{N^{(S-1)}}{\rho_{c}}\right) \frac{\partial n}{\partial t}+\frac{\partial u n}{\partial x}+\left(1-\frac{1}{\rho_{c}}\right) \frac{\partial N}{\partial t}- \\
& -\frac{n}{\rho_{c}} \frac{\partial N^{(S-1)}}{\partial t}+\frac{N}{\rho_{c}} \frac{\partial n^{(S-1)}}{\partial t}+\frac{1}{\rho_{c}} \frac{\partial(n N)^{(S-1)}}{\partial t}= \\
& =\frac{1}{P e} \frac{\partial}{\partial x} D \frac{\partial n}{\partial x}+\frac{D_{\delta}}{n_{0} u_{0}} \frac{1}{P} \frac{\partial P}{\partial x},
\end{aligned}
$$

where $P e=\frac{H_{0} u_{0}}{D}$ - Peclet number.

The boundary conditions of problem (1), (9) are written in the form

$$
\left\{\begin{array}{l}
n=1, N=0, t=0 \\
n=1, x=0 \\
n=0, x=1
\end{array}\right.
$$

Taking into account the clogging of the filter pores with gel particles, formulas (7) and (8) take the form

$$
\begin{aligned}
& m=\left[m_{1}+\delta\left(m_{0}-m_{1}\right)\right] \frac{m_{1}-\frac{N}{\rho_{c}}}{m_{1}}, \\
& k=\frac{A k_{0}}{S_{0}^{2}} \frac{m^{3}}{(1-m)^{2}}(1-\sqrt{\delta})^{3} .
\end{aligned}
$$

In a strict formulation, the solution of equations (1), (9) (11), (3) - (6) is difficult, since these equations would have to be solved jointly at each time layer. However, if in equations (3) - (6) the values $n$ and $N$ are taken as known (from the previous time layer), then the solution of the problem is greatly simplified, like the previous problem, and the filtration equations are solved independently of $n$ and $N$.

Equation (4) taking into account (11) takes the form

$$
\begin{aligned}
& q \frac{\partial \theta}{\partial t}+2 \frac{\partial m \theta}{\partial t}+\left(1-m_{0}\right) \frac{\partial m \delta}{\partial t}+q_{1} \theta- \\
& -q_{2}+m_{1} \theta \delta \frac{\partial q_{0}}{\partial t}+\frac{\partial u \theta}{\partial x}=\frac{\partial w}{\partial x} \\
& \frac{\partial w}{\partial x}=\frac{\mu_{0} \alpha_{\tau}}{x_{0}^{2}} \frac{\partial \theta}{\partial x}
\end{aligned}
$$


where

$$
\begin{aligned}
& q_{0}=1-\frac{N}{\rho_{c}}, \quad q=\delta\left[m_{1} q_{1}+\left(m_{0}-m_{1}\right) \delta\right], \\
& q_{1}=\lambda\left(2 \theta^{(S-1)}-\gamma \delta\right) q, q_{2}=\lambda q\left(\theta^{(S-1)}\right)^{2} .
\end{aligned}
$$

Equation (12) shows that even in such a simplified form, without additional assumptions, they cannot be reduced to a design scheme. Therefore, for the numerical solution of the problem in equation (12), we expand the derivatives 1 , 2 , excluding 3 , and performing quasilinearization, we obtain

$Q_{1} \frac{\partial \theta}{\partial t}+\left(Q_{2}+Q_{3}\right) \theta+a_{1} \delta-Q_{0}+\frac{\partial u \theta}{\partial x}=\frac{\partial w}{\partial x}$

where

$$
\begin{aligned}
& Q_{0}=2 \lambda a_{0} q_{0}\left(\theta^{(s-1)}\right)^{2}, Q_{1}=q+2 q_{0}\left(m_{1}+a_{0} \delta\right), \\
& Q_{2}=\left[2 m_{1}-\left(m_{1}+a_{0}\right) \delta\right] \frac{\partial q_{0}}{\partial t}, \\
& Q_{0}=2 \lambda q_{0}\left(2 \theta^{(s-1)}-\gamma \delta\right), a_{0}=m_{1}-m_{0} .
\end{aligned}
$$

Thus, equation (13) for the numerical solution of the filtering problem is preferable to direct approximation of equation (12).

For the numerical integration of these problems, the system of equations is approximated by a one-sided difference scheme with an accuracy of $O(\mathrm{~h})$, and the obtained algebraic equations are solved by the sweep method.

Let us consider a simpler model of the filtration process, when in the second equation of system (2) $u=$ const . In addition, it can be assumed that at a sufficient depth from the surface of the filter, the suspended particles do not settle, and the settled particles do not break off. Then $\alpha(x, t)$ is replaced by $\theta_{3}(t)=\theta(1, t)$ and we get

$$
\left\{\begin{array}{l}
\frac{\partial \theta}{\partial t}+a_{0} \frac{\partial \theta}{\partial x}+a_{1} \frac{\partial \delta}{\partial t}=D \frac{\partial^{2} \theta}{\partial x^{2}}-\frac{\partial \theta_{3}}{\partial t} \\
\frac{\partial \delta}{\partial t}=\lambda(\theta-\gamma \delta) \\
\theta=\theta_{0} e^{-b_{0} x}, \delta=0, t=0 \\
\theta=\theta_{0}, x=0 \\
\frac{\partial \theta}{\partial x}=-m a_{1} \frac{\partial \delta}{\partial t}, x=\eta_{2} .
\end{array}\right.
$$

Equation with boundary conditions (14) is a linear system with constant coefficients and, therefore, admits an analytical solution.

Note that a linear system admitting an analytical solution, on the one hand, serves as a model problem for debugging a numerical integration program, on the other hand, the obtained analytical solution can serve to solve the inverse problem of TP filtering.

We apply the Laplace transform to system (14) and obtain

$$
\begin{aligned}
& \theta^{\prime \prime}-\frac{a_{0}}{D} \theta^{\prime}-\frac{s\left(s+\lambda+\lambda_{1}\right)}{D\left(s+\lambda_{1}\right)} \theta=\frac{s \theta_{3}-\phi_{0}}{D}, \theta(0, s)=\frac{\theta_{0}}{s}, \\
& \theta^{\prime}\left(\eta_{2}, s\right)+\frac{b_{1} s}{s+\lambda_{1}} \theta\left(\eta_{2}, s\right)=0, \phi_{0}=\theta_{0} e^{-b_{0} x}, b_{1}=m \lambda a_{1} .
\end{aligned}
$$

Change the variables $\theta=u e^{k x}$ and get

$$
\begin{aligned}
& u^{\prime \prime}-v^{2} u=\phi(x), \\
& \left\{\begin{array}{l}
u(0, s)=\frac{\theta_{0}}{s}, \\
u^{\prime}\left(r_{2}, s\right)+\left(k+\frac{b_{1}}{s+\lambda_{1}}\right) u\left(r_{2}, s\right)=0,
\end{array}\right.
\end{aligned}
$$

where $v^{2}=k^{2}+\frac{s\left(s+b_{2}\right)}{D\left(s+\lambda_{1}\right)}, k=\frac{a_{0}}{2 D}, \quad b_{2}=\lambda+\lambda_{1}$.

We write the solution to homogeneous equation (15) in the form

$$
u=A e^{v x}+B e^{-v x} .
$$

Then the solution to the inhomogeneous equation is as follows:

$u=-C_{1} e^{k x}+C_{2} e^{-\left(b_{0}-k\right) x}+A_{0} e^{v x}+B_{0} e^{-v x}$,

where $C_{1}=\frac{D \theta_{3}\left(s+\lambda_{1}\right)}{s+b_{2}}, C_{2}=\frac{\theta_{0}}{D\left[v^{2}-\left(b_{0}-k\right)^{2}\right]}$.

Coefficients $A_{0}$ and $B_{0}$ are determined from (16):

$$
\begin{aligned}
& \frac{\theta_{0}}{s}=-q_{1}+q_{2}+A_{0}+B_{0}, x=0 \\
& -k C_{1} e^{k \eta_{2}}-\left(b_{0}-k\right) C_{2} e^{-\left(b_{0}-k\right) \eta_{2}}+\gamma\left(A_{0} e^{\gamma \eta_{2}}-B_{0} e^{-\gamma \eta_{2}}\right)+ \\
& +\left(k+\frac{b_{1}}{s+\lambda_{1}}\right)\left(-C_{1} e^{k \eta_{2}}+C_{2} e^{-\left(b_{0}-k\right) \eta_{2}}+A_{0} e^{\gamma \eta_{2}}+B_{0} e^{-\gamma \eta_{2}}\right)=0
\end{aligned}
$$

at $x=\eta_{2}$.

Solving them together, we find

$$
\begin{aligned}
& A_{0}=\frac{q_{0}+q_{0}^{\prime} q_{2}}{q_{1}+q_{2}}, \quad B_{0}=\frac{q_{0}^{\prime} q_{1}-q_{0}}{q_{1}+q_{2}} \\
& q_{0}=C_{1}\left(2 k+\frac{b_{1}}{s+\lambda_{1}}\right) e^{k \eta_{2}}+C_{2}\left(b_{0}-\frac{b_{1}}{s+\lambda_{1}}\right) e^{-\left(b_{0}-k\right) \eta_{2}}, \\
& q_{0}^{\prime}=C_{1}-C_{2}, q_{1}=C_{1}\left(\gamma+k+\frac{b_{1}}{s+\lambda_{1}}\right) e^{\gamma \eta_{2}}, \\
& q_{2}=C_{2}\left(\gamma-k-\frac{b_{2} s}{s+\lambda_{1}}\right) e^{-\gamma \eta_{2}} .
\end{aligned}
$$


As a first approximation, we will assume that

$b_{0}=0, \theta_{3}=0$.

This means that the initial distribution $\theta(x, 0)$ is constant. Assumption $\theta_{3}=0$ is valid, which follows from the fact that the suspension concentration at the exit through face $x=\eta_{2}$ is entirely determined by the boundary condition (16).

Note that the last condition (16) was obtained from the first two equations (14) under the assumption that the initial time is taken to be the time when the filtrate begins to exit through face $x=\eta_{2}$. Since the time to reach the filtrate of face $x=\eta_{2}$ is much less than the duration of the process, then, taking the indicated expression as the second condition, we do not contradict the condition of the problem.

Thus, the solution of the problem in images, subject to condition (18), takes the following form:

$$
\begin{aligned}
& u(x, s)=\frac{-D \theta_{0} b_{2} e^{k \eta_{2}}\left(s+\lambda_{1}\right) \frac{s h v x}{v}}{s\left(s+b_{2}\right)\left[c h v \eta_{2}+\left(k+\frac{b_{1}}{s+\lambda_{1}}\right) \frac{s h v x}{v}\right]}+ \\
& +\theta_{0}\left[\frac{1}{s}-\frac{s+\lambda_{1}}{s\left(s+b_{2}\right)}\right] \times \\
& \times \frac{\operatorname{chv}\left(x-\eta_{2}\right)-\left(k+\frac{b_{1}}{s+\lambda_{1}}\right) \frac{\operatorname{shv}\left(x-\eta_{2}\right)}{v}}{c h v \eta_{2}+\left(k+\frac{b_{1}}{s+\lambda_{1}}\right) \frac{s h v x}{v}}+ \\
& +\frac{\theta_{0}\left(s+\lambda_{1}\right)}{s\left(s+b_{2}\right)} .
\end{aligned}
$$

Solution (19) is the ratio of two generalized polynomials $\Phi(s) / \psi(s)$ with respect to $s$, and polynomial $\psi(s)$ does not contain a constant, i.e. all the conditions of the decomposition theorem are met, so it can be applied when passing from the image to the original:

$$
\begin{aligned}
& L^{-1}[\Phi(s) / \psi(s)]= \\
& =\sum_{n=1}^{\infty} \frac{\Phi(s)}{\psi^{\prime}(s)} e^{s_{n} t}
\end{aligned} .
$$

Thus, the analytical solution of the problem posed is finally obtained in the following form:

$$
\begin{aligned}
& \theta(x, t)=\theta_{0} e^{k x}\left\{\left(2 \lambda_{1}-\frac{\lambda_{1}-b_{2}}{b_{2}} e^{-b_{2} t}\right) \times\right. \\
& \times k c h k\left(x-\eta_{2}\right)-\left(k \lambda_{1}+b_{1}\right) \operatorname{shk}\left(x-\eta_{2}\right) \times \\
& \times\left(1+\lambda_{1}-\frac{\lambda_{1}-b_{2}}{b_{2}} e^{-b_{2} t}\right)+ \\
& \left.+D b_{2} e^{k \eta_{2}}\left(\lambda_{1}-\frac{\lambda_{1}-b_{2}}{b_{2}} e^{-b_{2} t}\right) \operatorname{shkx}\right\} \times \\
& \times \frac{1}{k \lambda_{1} c h k \eta_{2}+\left(k \lambda_{1}+b_{1}\right) \operatorname{shk} \eta_{2}}- \\
& \times\left[D b_{2} e^{k \eta_{2}} \operatorname{sh} x+\gamma c h \gamma \eta_{2}\left(x-\eta_{2}\right)\right]- \\
& -\sum_{i=1}^{2} \sum_{n=1}^{\infty} \frac{-\left(k s+k \lambda_{1}+b_{1}\right) \operatorname{sh} \gamma\left(x-\eta_{2}\right)}{\theta_{n i} D b_{2} e^{k \eta_{2}}\left(s_{n i}+\lambda_{1}\right) \sin \frac{\mu_{2} x}{\eta_{2}} e^{s_{n i} t} \times} \\
& \left.\times\left(s_{n i}\right) \sin \mu_{2}+\frac{\mu_{2}}{\eta_{2}} \cos \mu_{2}\right) \\
& \times\left(s_{n i}\right)=\frac{s_{n i}\left(s_{n i}+2 \lambda_{1}\right)}{2 D\left(s_{n i}+\lambda_{1}\right)} \times \\
& \left.\eta_{2} \sin \mu_{2}-\frac{1+\eta_{2}^{2}\left(k s_{n i}+\lambda_{1}+b_{1}\right)}{\mu_{2}} \cos \mu_{2}\right]
\end{aligned}
$$

And so, analytical solutions to the problems of TP of ionexchange filtration of a suspension have been obtained, with the help of which it is possible to determine changes in the concentration of liquid in the column of the filtering unit over time and to determine the main parameters of the process and their ranges of variation. predict and determine the main technological parameters of the object and their acceptable ranges of variation, as well as the obtained analytical solutions of the model problem can be used to solve the inverse filtering problem.

\section{CONCLUSION}

Using the obtained numerical and analytical solutions of the problems of the technological process of ion-exchange filtration of a suspension, it is possible to predict and determine the main technological parameters of the object and their acceptable ranges of variation.

The obtained analytical solutions of the model problem can be used to determine the main parameters of the technological process and their ranges of variation as an inverse problem, as well as to prove the adequacy of the developed mathematical models of the filtration process.

\section{REFERENCES}

1. D. Abdeli, and A. Seiden.High performance water treatment technology for the reservoir pressure maintenance at oil fields, Journal of Mechanical Engineering Research and Developments, Vol.41, pp. 66-81, 2018.

2. A. Raeini, M.J. Blunt, and B.Bijeljic. (2012) Modelling two-phase flow in porous media at the 
pore scale using the volume-offluid method,Journal of Computational Physics, Vol. 231, pp.5653-5668, 2012.

3. A. Safonyk, and A.Bomba.Mathematical modeling process of liquid filtration taking into account reverse influence of process characteristics on medium characteristics,International journal of applied mathematical research,Vol. 4, pp. 1-7, 2015.

4. B.Mukanova, and N. Glazyrina.Nonlinear Inverse Problem for an Ion-Exchange Filter Model:Numerical Recovery of Parameters, Mathematical Problems in Engineering, Vol. pp. 1-8, 2015.

5. A. Bomba, A.Safonyk, and V.N. Voloshchuk.Spatial modeling of multicomponent pollution removal for liquid treatment under identification of mass transfer coefficient,Mathematical Modeling and Computing,Vol. 5, pp. 108-118, 2018

6. F. Boyer, C. Lapuerta, S. Minjeaud, B. Piar, and M. Quintard. Cahn-Hilliard/Navier-Stokes Model for the Simulation of Three-Phase Flows,Transport in Porous Media. Vol. 82, pp. 463-483,2010.

7. C. Burkert, G. Barbosa, M. Mazutti, and F. Maugeri. Mathematical modeling and experimental breakthrough curves of cephalosporin $\mathrm{C}$ adsorption in a fixed-bed column,Process Biochemistry, Vol. 46, pp. 1270-1277, 2011.

8. L. Demeio, and D. Sani.A Proposed Model for Water-Salts Coupled Flow Transport Equations, Mathematical and Computer Modelling. Vol. 37, pp. 541-547, 2003.

9. S. Epoyan, A. Karahiaur, V. Volkov, and S. Babenko.Research into the influence of vertical drainage elements on the operational efficiency of rapid filters,Eastern-European Journal of Enterprise Technologies, Vol. 91, pp. 62-69, 2018.

10. G.Viccione,S. Evangelista, and G. Marinis. Experimental Analysis of the Hydraulic Performance of Filtering Cartridges in Drinking Water Networks, Water, Vol. 10, pp. 629, 2018.

11. V. Gitisa.Deep-bed filtration model with multistage deposition kinetics,ChemicalEngineering Journal. Vol. 163, pp. 78-85, 2010.

12. I. Golubev, Y. Karpova.Quality improvement of oilcontaminated wastewater, meant for injection into formation, using two-stage treatment technology, Journal of Ecological Engineering,Vol. 18, pp. 48-52, 2017.

13. R.C.Mamat, A. Ramli, A.M. Samad, A. Kasa, S.F.M. Razali, M.B.H. Che Omar. Stability Assessment of Embankment on Soft Soil Improved with Prefabricated Vertical Drains Using Empirical and Limit Equilibrium Approaches, International Journal of Advanced Trends in Computer Science and Engineering, Vol. 8, pp. 444-449, 2019.

14. Thangadurai N., S.B. Vinay Kumar, and C. Prasanna Kumar. Fertilizer Optimization by an Smart Soil Analyzer with a Soil Tester for Agriculture Applications, International Journal of Advanced Trends in Computer Science and Engineering, Vol. 8, pp. 3628-3631, 2019.

15. A. Delgado, N. Rojas, J. Oblitas, B. Andres, A. Huerta, and Ch. Carbajal. Water Quality Assessment using the Grey Clustering Analysis on a river of Taxco, Mexico, International Journal of Advanced
Trends in Computer Science and Engineering, Vol. 9, pp. 4717-4723, 2019.

16. M.H. Hamzah, A.M. Taib, S. Sharil, A.B. Ramli, and D.Z. AbangHasbollah. The Stability of Diaphragm Wall for Deep Excavation, International Journal of Advanced Trends in Computer Science and Engineering, Vol. 8, pp. 303-309, 2019.

17. N. Ravshanov, Sh.K. Daliev, and O. Tagaev. Numerical simulation of two aquarius Horizons, International Journal of Advanced Trends in Computer Science and Engineering, Vol. 9, pp. 65496554, August 2020.

18. A. Ab Aziz, A.R. Zulkefli, and M.A. Khasim. Finite Different Method and Differential Quadrature Method for Solving Burgers Equation, International Journal of Advanced Trends in Computer Science and Engineering, Vol. 8, pp. 5-8, 2019.

19. N. Ravshanov, U.M. Saidov, and D.I. Mutin. Modelling of the technological process of multiple filtering suspensions with multi-layered filter, $I O P$ Conference Series: Materials Science and Engineering, Vol. 537,April 2019.

20. N. Ravshanov, I.U. Shadmanov, and O.Ja. Kravets. Mathematical model for the study and prediction of a porous body thermal state, IOP Conference Series: Materials Science and Engineering, Vol. 537, pp. 1-8, April 2019.

21. 